\title{
Inhibition of aldose reductase ameliorates ethanol-induced steatosis in HepG2 cells
}

\author{
LONGXIN QIU $^{1,2}$, CHENGCHAO CAI $^{1}$, XIANGQIAN ZHAO $^{1}$, YAN FANG $^{1}$, \\ WEIBIAO TANG ${ }^{1}$ and CHANG GUO ${ }^{1,2}$ \\ ${ }^{1}$ Department of Biological Science and Technology, School of Life Sciences; \\ ${ }^{2}$ Fujian Key Laboratory of Preventive Veterinary Medicine and Biotechnology, \\ Longyan University, Longyan, Fujian 364012, P.R. China
}

Received February 1, 2016; Accepted January 1, 2017

DOI: $10.3892 / \mathrm{mmr} .2017 .6313$

\begin{abstract}
Aldose reductase (AR) expression is increased in liver tissue of patients with ethanol-induced liver disease. However, the exact role of AR in the development of ethanol-induced liver disease has yet to be elucidated. The present study aimed to determine the effect of an AR inhibitor on ethanol-induced steatosis in HepG2 cells and to identify possible underlying molecular mechanisms. Steatosis was induced in HepG 2 cells by stimulating cells with $100 \mathrm{mM}$ absolute ethanol for $48 \mathrm{~h}$. Oil Red $\mathrm{O}$ staining was used to detect the lipid droplet accumulation in cells. Western blot analyses were used to determine protein expression levels and reverse transcription-quantitative polymerase chain reaction was used to analyze mRNA expression levels. The results showed that AR protein expression was elevated in HepG 2 cells stimulated with ethanol. HepG2 cells exhibited marked improvement of ethanol-induced lipid accumulation following treatment with the AR inhibitor zopolrestat. Phosphorylation levels of 5' adenosine monophosphate-activated protein kinase (AMPK) were markedly higher, whereas the mRNA expression levels of sterol-regulatory element-binding protein (SREBP)-1c and fatty acid synthase (FAS) were significantly lower in zopolrestat-treated and ethanol-stimulated HepG2 cells compared with in untreated ethanol-stimulated HepG2 cells. In addition, zopolrestat inhibited the ethanol-induced expression of tumor necrosis factor (TNF)- $\alpha$. These results suggested that zopolrestat attenuated ethanol-induced steatosis by activating AMPK and subsequently inhibiting the expression of SREBP-1c and FAS, and by suppressing the expression of TNF- $\alpha$ in HepG2 cells.
\end{abstract}

Correspondence to: Professor Longxin Qiu, Department of Biological Science and Technology, School of Life Sciences, Longyan University, 1 North Dongxiao Road, Longyan, Fujian 364012, P.R. China

E-mail: qlongxin@tom.com

Key words: aldose reductase, steatosis, ethanol, AMP-activated protein kinase, sterol-regulatory element-binding protein-1c, tumor necrosis factor- $\alpha$

\section{Introduction}

Aldose reductase (AR; EC.1.1.1.21) belongs to the reduced coenzyme II-dependent aldo-keto reductase superfamily and it is the first enzyme in the polyol pathway of glucose metabolism (1). AR catalyzes the conversion of glucose to sorbitol with the aid of NADPH, and sorbitol dehydrogenase subsequently converts sorbitol to fructose, which can easily pass through cell membranes. AR was first discovered in the seminal vesicles. It is widely distributed in the brain, peripheral nerves, retina, heart, blood vessels, kidneys, and other tissues and organs (2). Alongside its extensive distribution, it exhibits a wide range of roles in various physiological processes, as has been demonstrated in in vivo models. For example, AR participates in energy production in sperm cells (1), serves a cytoprotective role during hyperosmotic stress (3), and is involved in detoxification mechanisms (4).

It has previously been reported that the polyol pathway, which involves AR, participates in the development of diabetic complications, in tissues severely affected by diabetes, such as the kidney, heart, retina and blood vessels (5). To the best of our knowledge, very few studies have explored the functions of AR in liver tissue. Under physiological circumstances, relatively little AR expression has been detected in the liver $(6,7)$; however, its expression is altered in disease states. Previous studies have demonstrated significant increases in AR expression levels in liver tissue from type II diabetic mice with hepatic steatosis (8) and mice with methionine-choline deficient diet-induced nonalcoholic fatty liver disease (9). The AR inhibitor zopolrestat significantly mitigated the steatosis and liver inflammation in type II diabetic mice (8) and methionine-choline deficient diet-induced mice (10). In addition, AR expression has been revealed to be significantly elevated in the liver tissue of patients with ethanol-induced liver disease $(11,12)$. However, the detailed mechanisms underlying the involvement of AR in the development of ethanol-induced liver disease have yet to be elucidated. The aim of the present study was to evaluate the effects of the AR inhibitor zopolrestat on ethanol-induced steatosis in HepG2 cells and to investigate the possible underlying molecular mechanisms. 


\section{Materials and methods}

Cell culture. Human hepatoma HepG2 cells were obtained from the Chinese Academy of Sciences Committee Type Culture Collection Cell Bank/CAS Shanghai Institute for Biologic Science Cell Resource Center (Shanghai, China) and cultured in Dulbecco's modified Eagle's medium supplemented with $10 \%$ fetal bovine serum (Hyclone; GE Healthcare Life Sciences, Logan, UT, USA), $100 \mathrm{U} / \mathrm{ml}$ penicillin and $100 \mu \mathrm{g} / \mathrm{ml}$ streptomycin. The cells were maintained in an incubator at $37^{\circ} \mathrm{C}$ in a $5 \% \mathrm{CO}_{2}$ atmosphere.

Oil red $O$ staining. HepG2 cells were plated in 6-well plates at a density of $\sim 4 \times 10^{5}$ cells/well. The following day, cells were stimulated with $100 \mathrm{mM}$ ethanol for $48 \mathrm{~h}$, and simultaneously treated with $50 \mu \mathrm{M}$ zopolrestat (Sigma-Aldrich; Merck KGaA, Darmstadt, Germany). A total of $48 \mathrm{~h}$ post-treatment, cells were washed with ice-cold PBS, fixed with $10 \%$ formalin, and stained with Oil Red $\mathrm{O}$ to detect lipid droplets in cells.

Reverse transcription-quantitative polymerase chain reaction (RT-qPCR). Total RNA was extracted from cells using TriPure Isolation Reagent (Roche Applied Science, Mannheim, Germany), according to the manufacturer's protocol. Total RNA was reverse transcribed into cDNA with the RevertAid First Strand cDNA Synthesis kit (Thermo Fisher Scientific, Inc., Waltham, MA, USA) according to the manufacturer's instructions. The primer sequences for hepatic tumor necrosis factor (TNF)- $\alpha$, transforming growth factor (TGF)- $\beta 1$, interleukin (IL)-6, sterol regulatory element binding protein (SREBP)-1c, fatty acid synthase (FAS), peroxisome proliferator-activated receptor (PPAR) $\alpha$, acyl-CoA oxidase (ACO) and carnitine palmitoyltransferase (CPT)-1 are presented in Table I. qPCR was conducted using the FastStart Universal SYBR-Green Master (Rox; Roche Applied Science). The reaction was run at $95^{\circ} \mathrm{C}$ for $10 \mathrm{~min}$, followed by 35 cycles at $95^{\circ} \mathrm{C}$ for $15 \mathrm{sec}, 53-60^{\circ} \mathrm{C}$ for $30 \mathrm{sec}, 72^{\circ} \mathrm{C}$ for $30 \mathrm{sec}$ and a final extension at $72^{\circ} \mathrm{C}$ for $10 \mathrm{~min}$. The relative expression of each gene was normalized to GAPDH and was calculated using the comparative $\mathrm{Cq}$ method $(\Delta \Delta \mathrm{Cq})(13)$.

Western blot analysis. Whole cell extracts were prepared by dissolution of cell pellets in ice-cold radioimmunoprecipitation assay lysis buffer (1\% Triton X-100, 50 mM HEPES, pH 7.5, $150 \mathrm{mM} \mathrm{NaCl}, 1 \mathrm{mM}$ EDTA, $10 \%$ glycerin, $10 \mathrm{mM}$ $\mathrm{Na}_{4} \mathrm{P}_{2} \mathrm{O}_{7}, 20 \mathrm{mM}$ glycerophosphate, $10 \mathrm{mM} \mathrm{NaF}, 10 \mathrm{mM}$ sodium orthovanadate and proteinase inhibitor mixture) until the cells were completely lysed. Following centrifugation at $12,000 \times \mathrm{g}, 4^{\circ} \mathrm{C}$ for $5 \mathrm{~min}$, supernatants were collected and stored at $-80^{\circ} \mathrm{C}$ before use. The protein concentrations of the extracts were measured using a bicinchoninic acid protein assay kit (Beyotime Institute of Biotechnology, Haimen, China). Equal amounts of extracted protein samples $(40 \mu \mathrm{g})$ were separated by $12 \%$ SDS-PAGE and transferred onto a polyvinylidene difluoride membrane (EMD Millipore, Billerica, MA, USA). Membranes were blocked with 5\% non-fat milk in $0.1 \%$ Tween-20 TBS for $1 \mathrm{~h}$ at room temperature and incubated with primary antibodies in TBS- $0.1 \%$ Tween-20 with $5 \%$ non-fat milk at $4{ }^{\circ} \mathrm{C}$ overnight. Primary antibodies used were: $5^{\prime}$ adenosine monophosphate-activated protein kinase $\alpha$ (AMPK $\alpha$; cat. no. \#2532; 1:1,000 dilution; Cell Signaling Technology, Inc., Danvers, MA, USA), phosphorylated (p)-AMPK $\alpha$ (cat. no. \#2535; 1:1,000 dilution; Cell Signaling Technology, Inc.), AR (cat. no. sc-17735; 1:500 dilution; Santa Cruz Biotechnology, Inc., Dallas, TX, USA) and $\beta$-actin (cat. no. A2228; 1:2,000 dilution; Sigma-Aldrich; Merck KGaA). After several washes with TBS-0.1\% Tween-20, the membranes were incubated with horseradish peroxidase-conjugated anti-rabbit or anti-goat immunoglobulin $\mathrm{G}$ secondary antibodies (dilution 1:2,000; cat. nos. AP307P and AB324P, respectively; Merck KGaA) in TBS-0.1\% Tween-20 with $5 \%$ non-fat milk. The bands were visualized using the SuperSignal Chemiluminescent Substrate kit (Beyotime Institute of Biotechnology). Densitometric analysis of protein bands was performed using ImageJ v. 1.40 software (National Institutes of Health, Bethesda, MD, USA).

Statistical analysis. The statistical significance of the difference between groups was assessed by Student's t-test for pair-wise comparisons or one-way analysis of variance, followed by a post hoc Bonferroni's test for multiple comparisons. Data are expressed as the mean \pm standard error of the mean. $\mathrm{P}<0.05$ was considered to indicate a statistically significant difference. The analysis was performed using GraphPad Prism software version 5.0 (GraphPad Software, Inc., La Jolla, CA, USA).

\section{Results}

$A R$ is induced following ethanol stimulation, whereas $A R$ inhibition attenuates ethanol-induced hepatic steatosis. Previous studies have demonstrated that stimulating cells with ethanol may induce hepatic steatosis $(14,15)$. In the present study, protein expression levels of AR were assessed in HepG2 cells stimulated with $100 \mathrm{mM}$ ethanol. AR protein expression in cells stimulated with ethanol for $36 \mathrm{~h}$ was $\sim 2.1 \mathrm{x}$ higher $(\mathrm{P}<0.05)$ compared with in control cells without ethanol stimulation (Fig. 1). To further investigate the role of AR in the development of ethanol-induced steatosis, AR activity was inhibited via treatment with the AR inhibitor zopolrestat $(50 \mu \mathrm{M})$. Oil Red O staining revealed a marked lipid accumulation in ethanol-stimulated HepG2 cells, whereas control cells without ethanol stimulation did not exhibit steatosis (Fig. 2). When AR activity was inhibited, lipid accumulation in ethanol-stimulated cells was markedly attenuated. These findings suggested that AR is involved in the development of ethanol-induced steatosis in hepatocytes.

AR inhibition activates AMPK andmitigates the ethanol-induced elevation of SREBP-1c and FAS MRNA expression. AMPK inactivation has been reported during the development of ethanol-induced hepatic steatosis $(16,17)$. SREBP-1c is a transcription factor regulated by AMPK, which modulates the expression of several lipogenic enzymes, including FAS $(18,19)$. To investigate the role AR serves in the development of ethanol-induced steatosis, the effect of AR inhibition on AMPK activity and on SREBP-1c and FAS mRNA expression was assessed in ethanol-stimulated HepG2 cells. Treatment with $100 \mathrm{mM}$ ethanol resulted in a significant reduction in AMPK phosphorylation in HepG2 cells; the reduction in p-AMPK levels was markedly attenuated when ethanol-stimulated cells 
Table I. Primer sequences used for mRNA amplification by reverse transcription quantitative polymerase chain reaction.

\begin{tabular}{lll}
\hline Gene & \multicolumn{1}{c}{ Forward primer } & \multicolumn{1}{c}{ Reverse primer } \\
\hline GAPDH & 5'-ACCCACTCCTCCACCTTTG-3' & 5'-CTCTTGTGCTCTTGCTGGG-3' \\
SREBP-1c & 5'-CGACATCGAAGACATGCTTCAG-3' & 5'-GGAAGGCTTCAAGAGAGGAGC-3' \\
FAS & 5'-GACATCGTCCATTCGTTTGTG-3' & 5'-CGGATCACCTTCTTGAGCTCC-3' \\
IL-6 & 5'-AAAAGTCCTGATCCAGTTC-3' & 5'-GAGATGAGTTGTCATGTCC-3' \\
TGF- $\beta 1$ & 5'-CCGAGAAGCGGTACCTGAAC-3' & 5'-GAGGTATCGCCAGGAATTGTTG-3' \\
TNF- $\alpha$ & 5'-CCAGACCAAGGTCAACCTC-3' & 5'-CCAGATAGATGGGCTCATACC-3' \\
PPAR $\alpha$ & 5'-GCAGAAACCCAGAACTCAGC-3' & 5'-ATGGCCCAGTGTAAGAAACG-3' \\
ACO & 5'-TCTGTTGACCTTGTTCGAGCAA-3' & 5'-CAAGCACAGAGCCAAGTGTCAC-3' \\
CPT-1 & 5'-ACAGTCGGTGAGGCCTCTTATGAA-3' & 5'-TCTTGCTGCCTGAATGTGAGTTGG-3'
\end{tabular}

SREBP, sterol regulatory element binding protein; FAS, fatty acid synthase; IL, interleukin; TGF, transforming growth factor; TNF, tumor necrosis factor; PPAR, peroxisome proliferator-activated receptor; ACO, acyl-CoA oxidase; CPT, carnitine palmitoyltransferase.

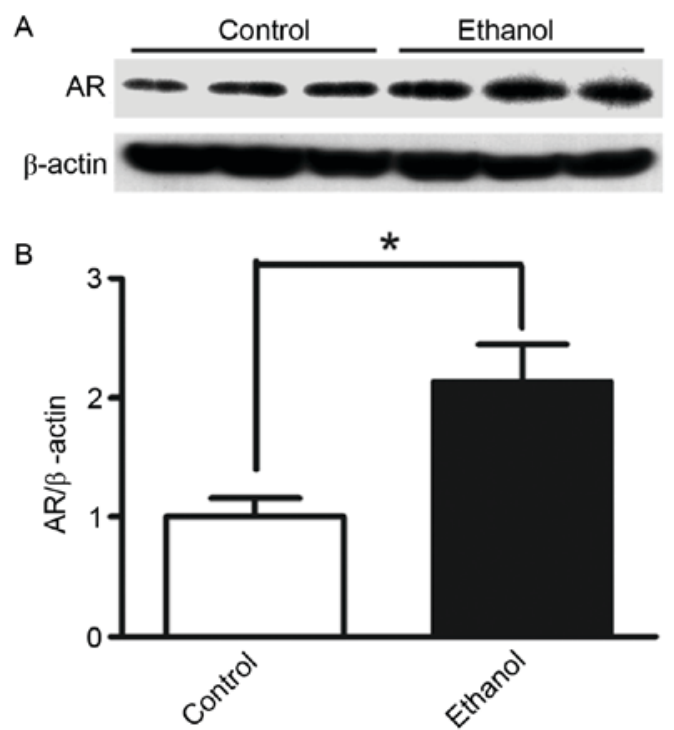

Figure 1. AR expression is potentiated in ethanol-stimulated HepG2 cells. (A) Representative western blot demonstrates the induction of AR protein expression in ethanol-stimulated hepatocytes. (B) Results of the densitometric analysis of the protein bands are presented as fold increase over the control cells without ethanol stimulation $(n=3)$. Data are expressed as the mean \pm standard error of the mean. " $\mathrm{P}<0.05$ vs. the control group. AR, aldose reductase.

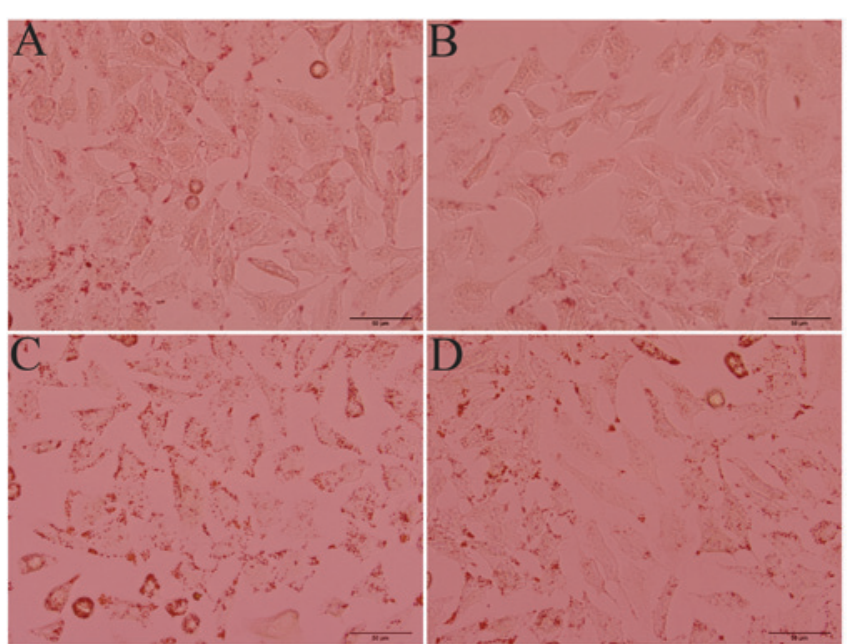

Figure 2. Treatment with the aldose reductase inhibitor zopolrestat ameliorated the ethanol-induced steatosis in HepG2 cells. Photomicrographs (400x magnification) of Oil Red O staining of (A) control cells without ethanol stimulation (B) control cells treated with zopolrestat (C) ethanol-stimulated cells and (D) ethanol-stimulated cells treated with zopolrestat. Images are representative of four separate experiments. were treated withzopolrestat (Fig. 3A). In addition, treatment with ethanol caused a significant increase in SREBP-1c mRNA expression levels compared with in untreated cells; the increase was significantly attenuated by zopolrestat treatment $(\mathrm{P}<0.05)$. FAS mRNA expression levels were significantly reduced in ethanol-stimulated cells treated with zopolrestat compared with in cells that didn't receive the AR inhibitor (Fig. 3B). Furthermore, the effect of AR inhibition on PPAR $\alpha$, a transcription factor that regulates several enzymes involved in fatty acid oxidation, such as ACO and CPT-1, was investigated $(20,21)$. AR inhibition did not alter the mRNA expression levels of PPAR $\alpha$, ACO or CPT-1 (Fig. 3C). Taken together, the present results suggested that the inhibition of AR may ameliorate ethanol-induced steatosis in HepG2 cells through activating AMPK and inhibiting SREBP-1c-regulated lipogenesis.
$A R$ inhibition attenuates the ethanol-induced elevation of $T N F-\alpha$ mRNA expression. TNF- $\alpha$ has been reported to serve a pivotal role in the development of ethanol-induced hepatic steatosis $(22,23)$. In the present study, mRNA expression levels of proinflammatory cytokines, including TNF- $\alpha$, IL-6 and TGF- $\beta 1$, were investigated. Cells stimulated with ethanol exhibited significantly elevated TNF- $\alpha$ mRNA expression levels compared with in unstimulated cells (Fig. 4), whereas treatment with zopolrestat significantly attenuated the ethanol-induced increase in TNF- $\alpha$ mRNA expression $(\mathrm{P}<0.05)$. Conversely, AR inhibition had no effect on IL-6 and TGF- $\beta 1$ expression. These results suggested that AR inhibition may affect the production of proinflammatory cytokines, such as TNF- $\alpha$, to prevent ethanol-induced steatosis in HepG2 cells. 

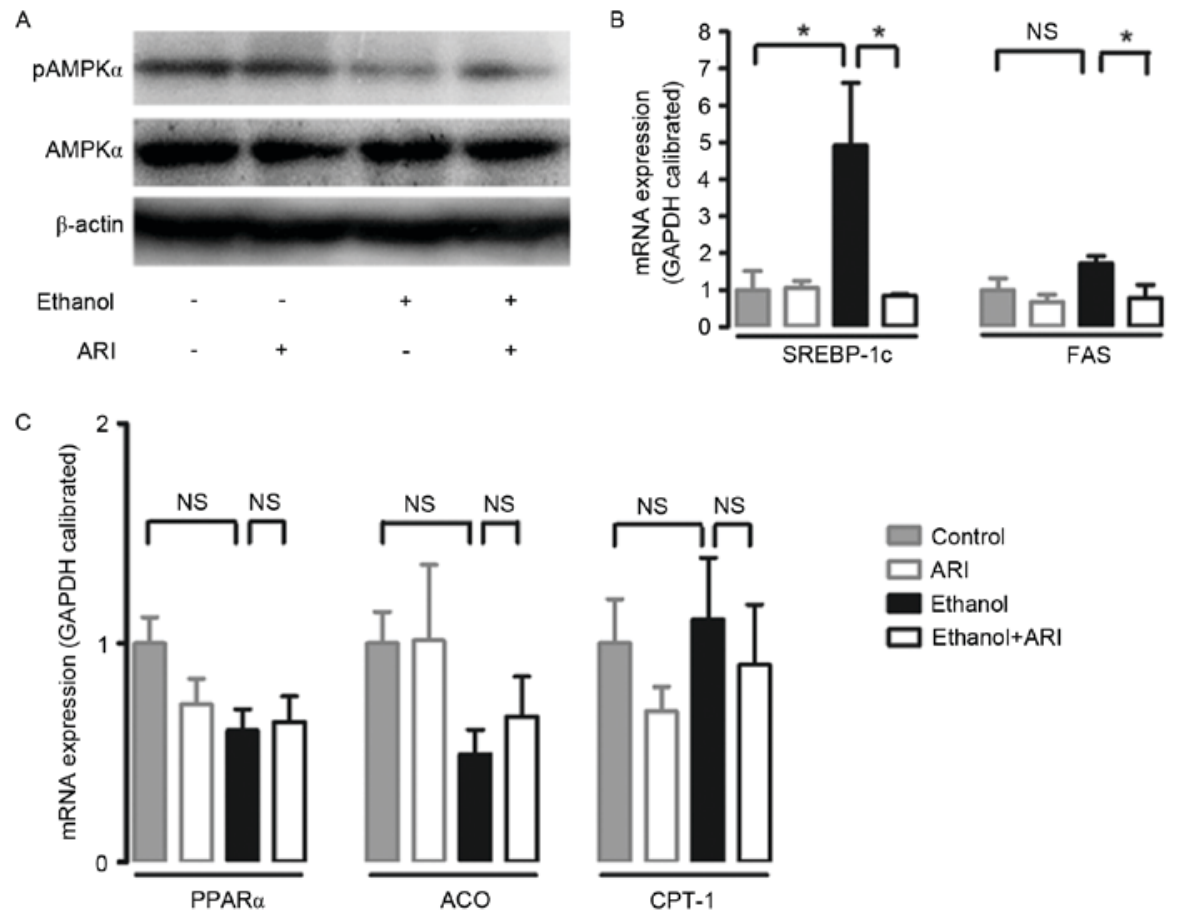

Figure 3. Effect of AR inhibition on AMPK activation and mRNA expression of lipid metabolism genes in ethanol-stimulated HepG2 cells. (A) Representative western blot demonstrates the activation of hepatic AMPK following AR inhibition in ethanol-stimulated cells. mRNA expression levels of (B) SREBP-1c and FAS, and (C) PPAR $\alpha$, ACO and CPT-1 were assessed by reverse transcription-quantitative polymerase chain reaction, standardized against an internal control $(\mathrm{GAPDH})$, and are presented as fold change over the control cells $(\mathrm{n}=3)$. Data are expressed as the mean \pm standard error of the mean. ${ }^{*} \mathrm{P}<0.05$. AR, aldose reductase; AMPK, 5 ' adenosine monophosphate-activated protein kinase; p, phosphorylated; SREBP, sterol regulatory element binding protein; FAS, fatty acid synthase; PPAR, peroxisome proliferator-activated receptor; ACO, acyl-CoA oxidase; CPT, carnitine palmitoyltransferase; NS, not significant.
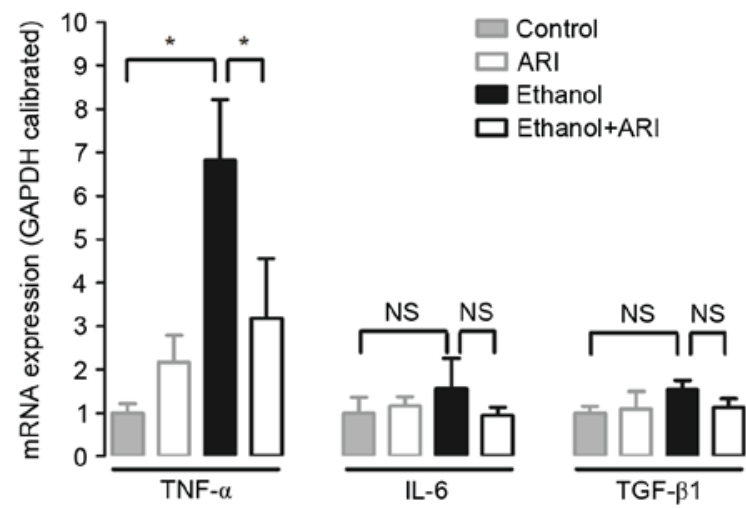

Figure 4. AR inhibition attenuated the ethanol-induced TNF- $\alpha$ mRNA overexpression in HepG2 cells. mRNA expression levels of TNF- $\alpha$, IL-6 and TGF- $\beta 1$ were assessed by reverse transcription-quantitative polymerase chain reaction, standardized against an internal control (GAPDH), and are presented as fold change over the control cells $(n=3)$. Data are expressed as the mean \pm standard error of the mean. ${ }^{*} \mathrm{P}<0.05$. AR, aldose reductase; $\mathrm{TNF}$, tumor necrosis factor; IL, interleukin; TGF, transforming growth factor; NS, not significant.

\section{Discussion}

Previous studies have suggested that AR may be involved in the development of non-alcoholic fatty liver disease, and that its inhibition may ameliorate hepatic steatosis (8-10). The present study demonstrated that ethanol induced an increase in AR expression, whereas the inhibition of AR markedly reduced lipid droplet accumulation in HepG 2 cells. These results suggested that $\mathrm{AR}$ may be involved in the development of alcoholic fatty liver disease, whereas its inhibition may ameliorate ethanol-induced hepatic steatosis.

AMPK is an enzyme that serves a role in cellular energy homeostasis, as its activation stimulates fatty acid oxidation and suppresses lipogenesis (24). AMPK activators have been reported to reduce the expression of SREBP-1c, a gene mainly associated with fat synthesis (25). In addition, SREBP-1c has been reported to act as a key regulator of hepatic lipid metabolism that modulates the transcription of various genes involved in hepatic triglyceride and fatty acid synthesis (18). Overexpression of SREBP-1c and one of its downstream genes, FAS, results in hepatic fat accumulation (18). Fatty acid synthesis appeared significantly potentiated in transgenic mice overexpressing SREBP-1c, and hepatic triglyceride levels were much increased (26). Acetaldehyde, which is a product of the hepatic metabolism of ethanol, was demonstrated to stimulate the overexpression of SREBP-1c in hepatocytes (27). Ethanol-induced SREBP-1c activation is one of the main causes of hepatic triglyceride accumulation in alcoholic fatty liver disease $(28,29)$. The results of the present study demonstrated that AR inhibition ameliorated the ethanol-induced AMPK inactivation and suppressed the ethanol-induced mRNA overexpression of SREBP-1c and FAS, thereby reducing hepatic lipid formation and mitigating hepatic steatosis. These results suggested that the AR-mediated dysregulation of the AMPK/SREBP-1c signaling pathway may contribute to the development of ethanol-induced hepatic steatosis.

The development of alcoholic liver diseases is closely associated with overexpression of several inflammatory cytokines, 
among which TNF- $\alpha$ holds a prominent role (23). Previous studies have reported that TNF- $\alpha$ expression is potentiated in patients with alcoholic liver diseases $(23,30)$. TNF- $\alpha$ induces lipid accumulation and promotes inflammatory and apoptotic processes in hepatocytes (31). The present study revealed that AR inhibition significantly reduced TNF- $\alpha$ mRNA expression levels. These results suggested that the amelioration of ethanol-induced hepatic steatosis achieved via AR inhibition may be partially attributed to the inhibition of ethanol-induced TNF- $\alpha$ overexpression.

In conclusion, in the present study, AR inhibition significantly mitigated the ethanol-induced lipid dysregulation in HepG2 cells, through the activation of AMPK and suppression of SREBP-1c and FAS. In addition, the AR inhibitor suppressed ethanol-induced TNF- $\alpha$ overexpression in hepatocytes. These results suggested that AR inhibition may improve ethanol-induced hepatic steatosis, whereas AR inhibitors may have potential as alternative therapeutic strategies for the treatment of alcoholic fatty liver diseases.

\section{Acknowledgements}

The present study was partly supported by the Training Program of Fujian Excellent Talents in University (grant no. MJR201558) and the Science Planning Program of Longyan University (grant no. LG2014012).

\section{References}

1. Hers HG: Aldose reductase. Biochim Biophys Acta 37: 120-126, 1960 (In French).

2. Clements RS Jr: The polyol pathway. A historical review. Drugs 32 (Suppl 2): S3-S5, 1986.

3. Ferraris JD, Williams CK, Jung KY, Bedford JJ, Burg MB and García-Pérez A: ORE, a eukaryotic minimal essential osmotic response element. The aldose reductase gene in hyperosmotic stress. J Biol Chem 271: 18318-18321, 1996.

4. Yabe-Nishimura C: Aldose reductase in glucose toxicity: A potential target for the prevention of diabetic complications. Pharmacol Rev 50: 21-33, 1998.

5. Nishimura-Yabe C: Aldose reductase in the polyol pathway: A potential target for the therapeutic intervention of diabetic complications. Nihon Yakurigaku Zasshi 111: 137-145, 1998 (In Japanese).

6. Clements RS Jr, Weaver JP and Winegrad AI: The distribution of polyol: NADP oxidoreductase in mammalian tissues. Biochem Biophys Res Commun 37: 347-353, 1969.

7. Markus HB, Raducha M and Harris H: Tissue distribution of mammalian aldose reductase and related enzymes. Biochem Med 29: 31-45, 1983.

8. Qiu L, Lin J, Xu F, Gao Y, Zhang C, Liu Y, Luo Y and Yang JY: Inhibition of aldose reductase activates hepatic peroxisome proliferator-activated receptor- $\alpha$ and ameliorates hepatosteatosis in diabetic db/db mice. Exp Diabetes Res 2012: 789730, 2012.

9. Qiu L, Lin J, Ying M, Chen W, Yang J, Deng T, Chen J, Shi D and Yang JY: Aldose reductase is involved in the development of murine diet-induced nonalcoholic steatohepatitis. PLoS One 8: e73591, 2013.

10. Chen T, Shi D, Chen J, Yang Y, Qiu M, Wang W and Qiu L: Inhibition of aldose reductase ameliorates diet-induced nonalcoholic steatohepatitis in mice via modulating the phosphorylation of hepatic peroxisome proliferator-activated receptor $\alpha$. Mol Med Rep 11: 303-308, 2015.

11. Brown KE, Broadhurst KA, Mathahs MM, Kladney RD, Fimmel CJ, Srivastava SK and Brunt EM: Immunodetection of aldose reductase in normal and diseased human liver. Histol Histopathol 20: 429-436, 2005.
12. O'Connor T, Ireland LS, Harrison DJ and Hayes JD: Major differences exist in the function and tissue-specific expression of human aflatoxin B1 aldehyde reductase and the principal human aldo-keto reductase AKR1 family members. Biochem J 343 Pt 2: 487-504, 1999.

13. Livak KJ and Schmittgen TD: Analysis of relative gene expression data using real-time quantitative PCR and the 2(-Delta Delta C(T)) Method. Methods 25: 402-408, 2001.

14. Keegan A, Martini R and Batey R: Ethanol-related liver injury in the rat: A model of steatosis, inflammation and pericentral fibrosis. J Hepatol 23: 591-600, 1995.

15. Altamirano $\mathrm{J}$ and Bataller R: Alcoholic liver disease: Pathogenesis and new targets for therapy. Nat Rev Gastroenterol Hepatol 8: 491-501, 2011.

16. Sid B, Verrax J and Calderon PB: Role of AMPK activation in oxidative cell damage: Implications for alcohol-induced liver disease. Biochem Pharmacol 86: 200-209, 2013.

17. You M, Matsumoto M, Pacold CM, Cho WK and Crabb DW: The role of AMP-activated protein kinase in the action of ethanol in the liver. Gastroenterology 127: 1798-1808, 2004.

18. Horton JD, Goldstein JL and Brown MS: SREBPs: Activators of the complete program of cholesterol and fatty acid synthesis in the liver. J Clin Invest 109: 1125-1131, 2002.

19. Roder K, Zhang L and Schweizer M: SREBP-1c mediates the retinoid-dependent increase in fatty acid synthase promoter activity in HepG2. FEBS Lett 581: 2715-2720, 2007.

20. Kong L, Ren W, Li W, Zhao S, Mi H, Wang R, Zhang Y, Wu W, Nan Y and Yu J: Activation of peroxisome proliferator activated receptor alpha ameliorates ethanol induced steatohepatitis in mice. Lipids Health Dis 10: 246, 2011.

21. Kota BP, Huang TH and Roufogalis BD: An overview on biological mechanisms of PPARs. Pharmacol Res 51: 85-94, 2005.

22. Thiele DL: Tumor necrosis factor, the acute phase response and the pathogenesis of alcoholic liver disease. Hepatology 9: 497-499, 1989.

23. Kawaratani H, Tsujimoto T, Douhara A, Takaya H, Moriya K, Namisaki T, Noguchi R, Yoshiji H, Fujimoto M and Fukui H: The effect of inflammatory cytokines in alcoholic liver disease. Mediators Inflamm 2013: 495156, 2013.

24. Viollet B, Guigas B, Leclerc J, Hébrard S, Lantier L, Mounier R, Andreelli F and Foretz M: AMP-activated protein kinase in the regulation of hepatic energy metabolism: From physiology to therapeutic perspectives. Acta Physiol (Oxf) 196: 81-98, 2009.

25. Tomita K, Tamiya G, Ando S, Kitamura N, Koizumi H, Kato S, Horie Y, Kaneko T, Azuma T, Nagata H, et al: AICAR, an AMPK activator, has protective effects on alcohol-induced fatty liver in rats. Alcohol Clin Exp Res 29 (12 Suppl): S240-S245, 2005.

26. Shimano H, Horton JD, Shimomura I, Hammer RE, Brown MS and Goldstein JL: Isoform 1c of sterol regulatory element binding protein is less active than isoform la in livers of transgenic mice and in cultured cells. J Clin Invest 99: 846-854, 1997.

27. Lluis JM, Colell A, García-Ruiz C, Kaplowitz N and Fernández-Checa JC: Acetaldehyde impairs mitochondrial glutathione transport in HepG2 cells through endoplasmic reticulum stress. Gastroenterology 124: 708-724, 2003.

28. You M, Fischer M, Deeg MA and Crabb DW: Ethanol induces fatty acid synthesis pathways by activation of sterol regulatory element-binding protein (SREBP). J Biol Chem 277: 29342-29347, 2002.

29. You M and Crabb DW: Recent advances in alcoholic liver disease II. Minireview: Molecular mechanisms of alcoholic fatty liver. Am J Physiol Gastrointest Liver Physiol 287: G1-G6, 2004.

30. McClain CJ and Cohen DA: Increased tumor necrosis factor production by monocytes in alcoholic hepatitis. Hepatology 9: 349-351, 1989.

31. Endo M, Masaki T, Seike M and Yoshimatsu H: TNF-alpha induces hepatic steatosis in mice by enhancing gene expression of sterol regulatory element binding protein-1c (SREBP-1c). Exp Biol Med (Maywood) 232: 614-621, 2007. 